\title{
Nutzen der Labormedizin
}

\author{
Andreas R. Huber ${ }^{\mathrm{a}}$, Martin Risch ${ }^{\mathrm{b}}$ \\ ${ }^{a}$ Prof. Dr. med., Past-Präsident SULM, ${ }^{b}$ Dr. med., Präsident Schweizerische Union für Labormedizin (SULM)
}

Alles, was wir im Medizinalbereich unternehmen, soll für den einzelnen Patienten, das Patientenkollektiv oder auch die Gesellschaft Nutzen bringen. Im Blut zirkulierende Biomarker erlauben mittlerweile eine eindrücklich tiefe und präzise Einsicht in die Stoffwechselvorgänge im menschlichen Körper, in Gesundheit und eben auch bei krankhaften Zuständen. Mittlerweile sind wir in der Lage, sehr spezifisch die Konzentrationen von Molekülen verschiedenster Art nachzuweisen. Zudem ist die Gewinnung von Probenmaterial meist nur minimal invasiv (Blutentnahme) oder kann durch Abstriche aus Körperteilen (Wunde, Schleimhaut) einfach gewonnen werden. Wenige Zellen, ja

\section{So sind die weitherum bekannten Schnelltests oft von ungenügender Qualität.}

auch nur Spuren, erlauben es, durch Analyse der DNA genetische Merkmale (in der Forensik) oder genetische Attribute bei genetischen Erkrankungen festzuhalten. In den letzten Jahren war die Entwicklung rasant. Sie lässt sich dem Gebiet der -omics zuordnen. Darunter Genomics, Proteomics, aber auch Metabolomics. Durch neue Methoden können in diesen drei Molekülklassen aus äusserst wenig Probenmaterial mehrere Substanzen, Gene oder Metaboliten recht kostengünstig nachgewiesen werden.

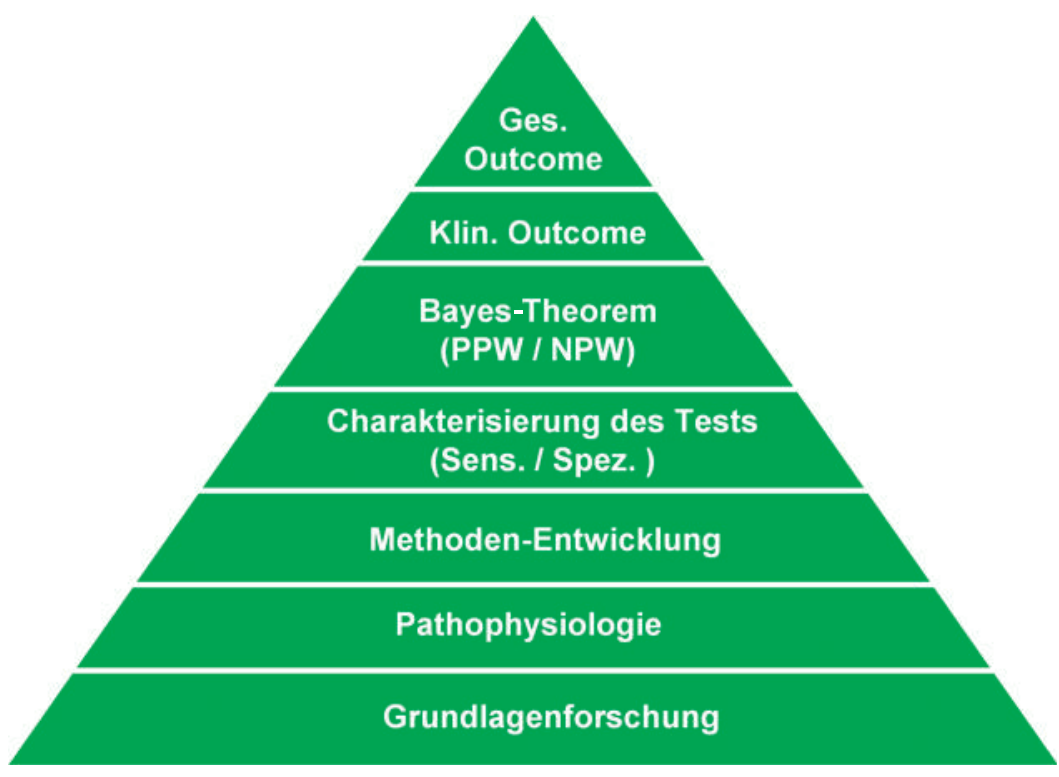

Abbildung 1: Entwicklungspyramide von Labortests.

(Bild: zVg vom Autor)

\section{Entwicklung von labormedizinischen Tests}

Abbildung 1 zeigt die Entwicklungspyramide von Labortests, die darlegt, wie ein Labortest von der Grundlagenforschung bis zum Outcome für die Gesellschaft entwickelt wird. Laborseitig wesentlich ist, eine robuste gute Methode zu entwickeln, die dann an einem Krankheitskollektiv mit gesunden Kontrollen getestet wird, worauf sich Sensitivitäten und Spezifitäten ableiten lassen. Dies ist klinisch allerdings wenig wertvoll, da die Patienten sich nicht als gesund oder krank präsentieren, sondern an oft unspezifischen Symptomen und Beschwerden leiden. Deshalb sind jeweils weitere Studien nötig, woraus sich dann die positive oder negative Wahrscheinlichkeit für ein Individuum mit entsprechenden Symptomen oder für ein Kollektiv mit entsprechender Prävalenz ableiten lässt. Je höher diese Werte sind, desto besser ist ein Test. Letztlich aber zählt der Outcome primär für das erkrankte Individuum, aber auch, wenn die ökonomischen und gesellschaft-

\section{Nutzen der Labormedizin}

\section{am Swiss MedLab Kongress 2016}

Vom 13. bis 16. Juni 2016 findet der Swiss MedLab Kongress in Bern statt. Es ist der einzige Schweizer Laborkongress, der den interdisziplinären Austausch unter allen Fachgebieten möglich macht. Dabei hat insbesondere der Fokus auf den «Nutzen der Labormedizin» ein starkes Gewicht.

Eingebunden in den Swiss MedLab Kongress bietet die jährliche gesundheitspolitische Tagung der SULM unter dem Titel «Nutzen der Labormedizin» einen breiten Überblick zum Stand der Forschung und Praxis.

Den Auftakt macht Dr. Stephan Sigrist mit seiner Keynote zu "Hacking Healthcare». Andreas Faller widmet sich im Anschluss dem Thema "Was bedeutet ein freiheitliches Gesundheitswesen für die Labormedizin?». Dr. Christoph Bosshard legt den Fokus unter dem Stichwort "Overuse" auf unnötige Behandlungen als Qualitätsproblem. Nationalrat Jean-François Steiert schildert den Nutzen der Labormedizin aus Sicht der nationalrätlichen Kommission für Soziale Sicherheit und Gesundheit. Die «Bedeutung der Labordiagnostik für die Krankenhausversorgung" beleuchtet Dr. Sabine Löffert. Prof. Andreas Huber berichtet über aktuelle Studien zu "Labor und Outcome» und Prof. Hans-Florian Zeilhofer schliesst mit dem Titel «Nutzen der Innovation für die Labormedizin».

Die folgende Podiumsdiskussion mit den Referenten und dem Publikum ermöglicht es, das Gehörte im Austausch zu vertiefen und präzisieren.

Weitere Informationen: www.sulm.ch/swissmedlab 


\begin{tabular}{|c|c|c|c|}
\hline Parameter & Topic & Bewertung & Quelle \\
\hline PCT & Infekt & Wertigkeit von PCT bei Infekten & [2] \\
\hline PCT & Harnwegsinfekt & Wertigkeit von РСТ bei speziellen Infekten & [3] \\
\hline PSA & Prostata CA & Konzentrationsabhängiges Screening mit Stufenschema & [6] \\
\hline BCR-ABL & $\mathrm{CML}$ & Steuerung der Therapie mit Tyrosinkinase-Blocker & [7] \\
\hline Copeptin & Hyponatriämie & Beurteilung des Verlaufs & [8] \\
\hline Mehrere Biomarker & Pneumonie & Verbessertes Management des Patienten in der Notfallstation & {$[11]$} \\
\hline Mehrere Biomarker & Herzinsuffizienz & Verbesserung der Hospitalisationsdauer & {$[12]$} \\
\hline PCT & Pneumonie & Bessere Behandlungsstrategie im Notfall und Spital & [13] \\
\hline Mehrere Biomarker & Mehrere Krankheiten & Übersichtsartikel & [14] \\
\hline
\end{tabular}

lichen Komponenten miteinbezogen werden, für die Gesellschaft.

\section{Outcome}

Im Labor geht es letztlich darum, aufzuzeigen, wie ein Labortest die ganze Diagnose- und Therapiekette günstig beeinflussen kann. In den letzten zehn Jahren sind mehrere bedeutende Outcome-Studien durchgeführt und publiziert worden. Jedes Outcome wird durch verschiedene Faktoren beeinflusst. Darunter die richtige Patientenselektion, die richtige Auswahl des Tests mit einer entsprechend hochqualifizierten Methode, Wahl des richtigen Zeitpunkts, korrekte Lagerung und Transport. In der Tabelle 1 finden sich einige kürzlich publizierte wichtige Studien, die den Wert von Laboranalysen belegen. Da Labortests relativ kostengünstig sind, ist auch das Kosten-Nutzen-Verhältnis sehr positiv. Wie mit allem gibt es auch Limitationen. So sind die weitherum bekannten Schnelltests oft von ungenügender Qualität und verursachen eine hohe Zahl an falsch positiven, aber auch falsch negativen Resultaten mit entsprechenden Folgekosten und auch Auswirkungen auf die Patienten. Es ist wichtig, dass der Anwender die Spezifitäten und Sensitivitäten der Tests kennt und entsprechend Resultate zu interpretieren weiss. Auch spielt es eine Rolle, wo der sogenannte Decision Point resp. der Cut-off zwischen gesund und krank gelegt wird. Wichtig ist, dass mit klinischen Scores oder aber
Prävalenzen Wahrscheinlichkeiten abgeschätzt werden, bevor definitive Diagnosen oder Ausschlüsse gemacht werden. Sicher sind auch biologische Variationen und präanalytische Faktoren einzubeziehen. Wir warnen vor einer Polypragmasie, d.h. das zu häufige und ungezielte Anwenden von Labortests. Grundlage ist immer die Fragestellung, die Indikation und im Anschluss eine differenzierte Beurteilung des Resultates, die Plausibilisierung.

\section{Zusammenfassung}

Innert den wenigen letzten Jahren haben sich die labordiagnostischen Möglichkeiten enorm weiterentwickelt. Immer schneller, immer besser und auch günstiger können Moleküle oder Molekülgruppen, Stoffwechselprodukte, Medikamente oder Gene nicht invasiv nachgewiesen werden. In der Literatur finden sich zunehmend eindrückliche Studien, die ein verbessertes Outcome durch Einsatz eines oder mehrerer Labortests belegen. Mittlerweile haben viele dieser Labortests ihren Weg in die offiziellen Guidelines und Algorithmen der verschiedenen Fachgesellschaften gefunden. Als Beispiele mögen gelten HbAlc, BNP, Troponine, PSA, Procalcitonin, Proadrenomedullin, Copeptin; der Einsatz dieser Tests aber bedingt eine gute Indikation sowie eine gute Interpretation mit Einbezug anderer Fakten, wie sie aus Anamnese, Status und anderen Abklärungen erhoben werden. 


\section{Literatur}

1 Foxman B. Urinary tract infection syndromes: occurrence, recurrence, bacteriology, risk factors, and disease burden. Infectious disease clinics of North America 2014;28(1):1-13.

2 Schuetz P, Albrich W, Mueller B. Procalcitonin for diagnosis of infection and guide to antibiotic decisions: past, present and future. BMC Med 2011;9:107.

3 van Nieuwkoop C, Bonten TN, van't Wout JW, et al. Procalcitonin reflects bacteremia and bacterial load in urosepsis syndrome: a prospective observational study. Critical care 2010; 14(6):R206.

4 Ottiger C, Schaer G, Huber AR. Time-course of quantitative urinary leukocytes and bacteria counts during antibiotic therapy in women with symptoms of urinary tract infection. Clin Chim Acta 2007; 379(1-2):36-41.

5 Drozdov D, Thomer A, Meili M, et al. Triple P study group. Procal citonin, pyuria and proadrenomedullin in the management of urinary tract infections - 'triple p in uti': study protocol for a randomized controlled trial. Trials. 2013;14:84.

6 Randazzo M, Beatrice J, Huber A, et al. A «PSA Pyramid» for Men with Initial Prostate-specific Antigen $<3 \mathrm{ng} / \mathrm{ml}$ : A Plea for Individualized Prostate Cancer Screening. Eur Urol. 2014 Apr 18. pii: S0302-283800318-2. doi: 10.1016/j.eururo.2014.04.005.

7 Soverini S, Hochhaus A, Nicolini FE et al. BCR-ABL kinase domain mutation analysis in chronic myeloid leukemia patients treated with tyrosine kinase inhibitors: recommendations from an expert panel on behalf of European LeukemiaNet. Blood 2011;118: 1208-15.

8 Nigro N, Müller B, Morgenthaler N, et al. The use of copeptin, the stable peptide of the vasopressin precursor, in the differential diagnosis of sodium imbalance in patients with acute diseases. Swiss Med Wkly. 2011;141:13270
9 Widmer D, Drozdov D, Rüegger K, et al. Effectiveness of Proadrenomedullin Enhanced CURB65 Score Algorithm in Patients with Community-Acquired Pneumonia in "Real Life», an Observational Quality Control Survey. J Clin Med 2014;3:267-79.

10 Schröder FH, Hugosson J, Roobol MJ, et al. Screening and prostate cancer mortality: results of the European Randomised Study of Screening for Prostate Cancer (ERSPC) at 13 years of follow-up. Lancet. 2014 Aug 6. pii: S0140-673660525-0. doi: 10.1016/S0140673660525-0

11 Sousa Da Silva RX, Dusemund F, Nickel C, Bingisser R, Huber A, Müller B, Albrich WC. Validation of a risk-based biomarker-enhanced scoring system for lower respiratory tract infections (OPTIMA I Basel) - An observational survey. International Journal of Clinical Medicine 2013;4:69-77.

12 Dusemund F, Steiner M, Vuilliomenet A et al. Multidisciplinary Assessment to Personalize Length of Stay in Acute Decompensated Heart Failure (OPTIMA II ADHF). J Clin Med Res 2012;4: 402-9.

13 Schuetz P, Christ-Crain M, Albrich W, Zimmerli W, Mueller B. Guidance of antibiotic therapy with procalcitonin in lower respiratory tract infections: Insights into the ProHOSP study. Virulence 2010;1:88-92.

14 Winzeler BF, Christ-Crain M. Hormone als Biomarker für Diagnose und Prognose. Praxis (Bern 1994). 2012;101:251-7. 